\title{
On Sandwich Theorem for Certain Subclasses of Symmetric Analytic Functions Associated with Noor Integral Operator
}

\author{
C. Selvaraj ${ }^{1}$, T. R. K. Kumar ${ }^{2}$ \\ ${ }^{1}$ Department of Mathematics, Presidency College, Chennai, Tamilnadu, India \\ ${ }^{2}$ Department of Mathematics, R. M. K. Engineering College, Tamilnadu, India
}

\begin{abstract}
In this paper, we obtain some interesting properties of differential subordination and superordination for the classes of symmetric analytic functions in the unit disk, by applying Noor integral operator. We investigate several sandwich theorems on basis of this theory.
\end{abstract}

Keywords: Convex functions, Differential subordination and superordination, Noor integral operator, Best dominant

\section{Introduction}

Let $\mathrm{H}(\mathrm{U})$ denote the class of analytic functions in the open unit disk $U=\{z:|z|<1\}$ and let $\mathrm{H}[a, 1]$ denote the subclass of the functions $f \in \mathrm{H}(\mathrm{U})$ of the form:

$$
f(z)=a+a_{1} z+a_{2} z^{2}+\cdots(a \in \mathrm{C})
$$

Also, let $A$ be the class of functions $f \in \mathrm{H}(\mathrm{U})$ of the form

$$
f(z)=z+\sum_{n=2}^{\infty} a_{n} z^{n}
$$

For two functions $f(z)$ given by (1) and

$$
g(z)=z+\sum_{n=2}^{\infty} b_{n} z^{n}
$$

The Hadamard product (or convolution) of $f$ and $g$ is defined by

$$
(f * g)(z)=z+\sum_{n=2}^{\infty} a_{n} b_{n} z^{n}=(g * f)(z) .
$$

Let $f, g \in \mathrm{H}(\mathrm{U})$, we say that the function $f$ is subordinate to $g$, if there exist a Schwarz function $w$, analytic in $\mathrm{U}$, with $w(0)=0$ and $|w(z)|<1(z \in \mathrm{U})$, such that $f(z)=g(w(z))$ for all $z \in \bigcup$.

This subordination is denoted by $f \prec g$ or $f(z) \prec g(z)$. It is well known that, if the function $g$ is univalent in $U$, then $f(z) \prec g(z)$ if and only if $f(0)=g(0)$ and $f(\mathrm{U}) \subset g(\mathrm{U})$.

Let $p(z), h(z) \in \mathrm{H}(\mathrm{U})$, and $\Phi(r, s, t ; z): \mathrm{C}^{3} \times \mathrm{U} \rightarrow \mathrm{C}$. If $\quad p(z) \quad$ and $\Phi\left(p(z), z p^{\prime}(z), z^{2} p^{\prime \prime}(z) ; z\right)$ are univalent functions, and if $p(z)$ satisfies the second-order superordination

$$
h(z) \prec \Phi\left(p(z), z p^{\prime}(z), z^{2} p^{\prime \prime}(z) ; z\right)
$$

then $p(z)$ is called to be a solution of the differential superordination (2). (If $f(z)$ is subordinatnate to $g(z)$, then $g(z)$ is called to be superordinate to $f(z))$. An analytic function $q(z)$ is called a subordinant if $q(z) \prec p(z)$ for all $p(z)$ satisfies (2). An univalent subordinant $\tilde{q}(z)$ that satisfies $q(z) \prec \tilde{q}(z)$ for all subordinants $q(z)$ of (2) is said to be the best subordinant.

Recently, Miller amd Mocanu [13] obtained conditions on $h(z), q(z)$ and $\Phi$ for which the following implication holds true:

$$
h(z) \prec \Phi\left(p(z), z p^{\prime}(z), z^{2} p^{\prime \prime}(z) ; z\right) \Rightarrow q(z) \prec p(z)
$$

Using these results, the authors in [3] considered certain classes of first- order differential superordinations, see also [7], as well as superordination-preserving integral operators [6]. Aouf et al. [3, 4], obtained sufficient conditions for certain normalized analytic functions $f(z)$ to satisfy

$$
q_{1}(z) \prec \frac{z f^{\prime}(z)}{f(z)} \prec q_{2}(z)
$$

where $q_{1}(z)$ and $q_{2}(z)$ are given univalent functions in $\mathrm{U}$ with $q_{1}(0)=1$ and $q_{2}(0)=1$.

In [18], Sakaguchi defined the class of starlike functions with respect to symmetrical points as follows:

Let $f \in \mathrm{A}$. Then $\mathrm{f}$ is said to be starlike with respect to symmetrical points in $U$ if, and only if,

$$
\mathrm{R} \frac{z f^{\prime}(z)}{f(z)-f(-z)}>0, \quad(z \in \bigcup) \text {. }
$$

Obviously, it forms a subclass of close-to-convex functions and hence univalent. Moreover, this class includes the class 


\section{International Journal of Science and Research (IJSR) \\ ISSN (Online): 2319-7064}

Index Copernicus Value (2013): 6.14 | Impact Factor (2015): 6.391

of convex functions and odd starlike functions with respect to the origin, see [18].

Let $\mathrm{A}$ denote by $D^{\alpha}: \mathrm{A} \rightarrow \mathrm{A}$ the operator defined by

$$
D^{\alpha} f(z)=\frac{z}{(1-z)^{\alpha+1}} * f(z) \quad(\alpha>-1)
$$

or equiavalently,

$$
D^{k} f(z)=\frac{z\left(z^{k-1} f(z)\right)^{(k)}}{k !} \quad k \in \mathrm{N}_{0}=0,1,2, \ldots
$$

where the symbol $(*)$ stands for the Hadamard product (or Convolution). We note that $D_{0} f(z)=f(z)$ and $D^{1} f(z)=z f^{\prime}(z)$. The operator $D^{k} f$ is called the Ruscheweyh derivative of $k t h$ order of $f$, see [17]. Analogous to $D^{k} f$, Noor [14] and Noor et al. [15] defined an integral operator $I_{k}: \mathrm{A} \rightarrow \mathrm{A}$ as follows.

Let $f_{k}(z)=\frac{z}{(1-z)^{k+1}}, k \in \mathrm{N}_{0}$, and let $f_{k}^{(\tau)}$ be defined such that

$$
f_{k}(z) * f_{k}^{(\tau)}(z)=\frac{z}{(1-z)^{2}}
$$

Then

$$
I_{k} f(z)=f_{k}(z) * f_{k}^{(\tau)}(z)=\left[\frac{z}{(1-z)^{k+1}}\right]^{\tau} * f(z) .
$$

From (3) it is easy to verify that

$$
z\left(I_{k+1} f(z)\right)^{\prime}=(k+1) I_{k} f(z)-k I_{k+1} f(z) \text {. }
$$

We note that $I_{0} f(z)=z f^{\prime}(z)$ and $I_{1} f(z)=f(z)$. The operator $I_{k} f(z)$ defined by (3) is called the Noor Integral operator of $k t h$ order of $f$, see [8]. Moreover, Liu [8] introduced some new subclasses of strongly starlike functions defined by using the Noor integral operator and studied their properties. Liu and Noor [9] investigated some interesting properties of the Noor integral operator.

Definition 1.1 A function $f \in \mathrm{A}$ is said to be in the class $\mathrm{B}^{\lambda, \mu}(A, B)$, if it satisfies the following subordination condition:

$$
(1-\lambda)\left[\frac{I_{k+1} f(z)-I_{k+1} f(-z)}{2 z}\right]^{\mu}+\lambda \frac{z\left\{I_{k} f(z)-I_{k} f(-z)\right\}}{I_{k+1} f(z)-I_{k+1} f(-z)}\left[\frac{I_{k+1} f(z)-I_{k+1} f(-z)}{2 z}\right]^{\mu} \prec \frac{1+A z}{1+B z}
$$

where and throughout this paper unless otherwise mention the parameters $\lambda, \mu, \mathrm{A}$ and $\mathrm{B}$ are constrained as follows:

$$
\lambda \in \mathrm{C}: \mathrm{R}(\mu)>0:-1 \leq B \leq 1, A \neq B, A \in R,
$$

and all powers are understood as principal values.

In this paper, we prove such results as subordination and superordination properties, convolution properties, distortion theorems, and inequality properties of the class $\mathrm{B}^{\lambda, \mu}(A, B)$. For interested readers see the work done by the authors [1, 5].

\section{Preliminary Results}

Definition 2.1 Let $Q$ be the set of all functions $f$ that are analytic and injective on $\overline{\mathrm{U}} \backslash E(f)$, where

$$
E(f)=\left\{\xi \in \partial \cup: \lim _{z \rightarrow \xi} f(z)=\infty\right\},
$$

and are such that $f^{\prime}(\xi) \neq 0$ for $\xi \in \partial \bigcup \backslash E(f)$.

To establish our main results we need the following Lemmas.

Lemma 2.1 (Miller and Mocanu [12, 13]). Let the function $h(z)$ be analytic and convex (univalent) in $\bigcup$ with $h(0)=1$. Suppose also that the function $\Phi(z)$ given $b$

$$
\Phi(z)=1+c_{1} z+c_{2} z^{2}+\ldots
$$

is analytic in $U$,

$$
\Phi(z)+\frac{z \Phi^{\prime}(z)}{\gamma} \prec h(z) \quad(z \in \mathrm{U}, \mathrm{R} \gamma \geq 0 ; \gamma \neq 0),
$$

then $\Phi(z) \prec \Psi(z)=\frac{\gamma}{z^{\gamma}} \int_{0}^{z} t^{\gamma-1} h(t) d t \prec h(z)$,

$(z \in U)$, and $\Psi(z)$ is the best dominant of (1).

Lemma 2.2 (Shanmugam et al. [19]). Let $\sigma \in \mathrm{C}, \eta \in \mathrm{C}^{*}=\mathrm{C} \backslash 0$ and let $q$ be a convex univalent function in $\bigcup$ with

$$
\mathrm{R}\left(1+\frac{z q^{\prime \prime}(z)}{q^{\prime}(z)}\right)>\max \left\{0 ;-\mathrm{R}\left(\frac{\sigma}{\eta}\right)\right\}, \quad(z \in \mathrm{U}),
$$

If $p$ is analytic in $\bigcup$ and

$$
\sigma p(z)+\eta z p^{\prime}(z) \prec \sigma q(z)+\eta z q^{\prime}(z)
$$

then $p(z) \prec q(z)$, and $q$ is the best dominant of (3).

Lemma 2.3 ([13]). let $q(z)$ be a convex univalent function in $\bigcup$ and let $m \in \mathrm{C}, m>0$. Further assume that $\mathrm{R} m>0$. If $g(z) \in \mathrm{H}[q(0), 1] \cap Q$, and

$$
g(z)+m z q^{\prime}(z) \prec g(z)+m z g^{\prime}(z),
$$

implies $q(z) \prec g(z)$, and $q(z)$ is the best subordinant.

Lemma 2.4 ([10]). let $F$ be a analytic and convex in U. If $f, g \in \mathrm{A}$ and $f, g \prec F$ Then

$$
\lambda f+(1-\lambda) g \prec F, \quad(0 \leq \lambda \leq 1) .
$$

Lemma 2.5 ([16]). let $f(z)=1+\sum_{n=1}^{\infty} a_{n} z^{n}$ be analytic 


\section{International Journal of Science and Research (IJSR) \\ ISSN (Online): 2319-7064}

Index Copernicus Value (2013): 6.14 | Impact Factor (2015): 6.391

and convex in $\mathrm{U}$ and $g(z)=1+\sum_{n=1}^{\infty} b_{n} z^{n}$ be analytic and convex in $U$. If $f(z) \prec g(z)$, then $\left|a_{n}\right|<\left|b_{1}\right|, \quad(n \in \mathrm{N})$.

\section{Main Results}

Theorem 3.1 Let $f(z) \in \mathrm{B}^{\lambda, \mu}(A, B)$ with $\mathrm{R} \lambda>0$. Then

$$
\begin{aligned}
{\left[\frac{I_{k+1} f(z)-I_{k+1} f(-z)}{2 z}\right]^{\mu} \prec \Psi(z) } & =\frac{\mu(k+1)}{\lambda} \int_{0}^{1} \frac{1+A z u}{1+B z u} u^{\frac{\mu(k+1)}{\lambda}-1} d u \\
& \prec \frac{1+A z}{1+B z}
\end{aligned}
$$

and $\Psi(z)$ is the best dominant.

Proof. Set

$$
\left[\frac{I_{k+1} f(z)-I_{k+1} f(-z)}{2 z}\right]^{\mu}=h(z), \quad(z \in \mathrm{U}) .(10)
$$

Then $h(z)$ is analytic in $U$ with $h(0)=1$. Logarithmic differentiation of (5) and simple computations yield

$$
=h(z)+\frac{\lambda}{\mu(k+1)} z h^{\prime}(z) \prec \frac{1+A z}{1+B z} .
$$

Applying Lemma 2.2 to (11) with $\gamma=\frac{\mu(n+1)}{\lambda}$, we have

$$
\begin{aligned}
& {\left[\frac{I_{k+1} f(z)-I_{k+1} f(-z)}{2 z}\right]^{\mu} \prec \Psi(z)} \\
& =\frac{\mu(k+1)}{\lambda} z^{-\frac{\mu(k+1)}{\lambda}} \int_{0}^{z} \frac{1+A t}{1+B t} t^{\frac{\mu(k+1)}{\lambda}-1} d t \\
& =\frac{\mu(k+1)}{\lambda} \int_{0}^{1} \frac{1+A z u}{1+B z u} u^{\frac{\mu(n+1)}{\lambda}-1} d u \prec \frac{1+A z}{1+B z},
\end{aligned}
$$

and $\Psi(z)$ is the best dominant. This completes the proof.

Theorem 3.2 Let $q(z)$ be univalent in $\mathrm{U}, \lambda \in \mathrm{C}^{*}$. Suppose also that $q(z)$ satisfies the following inequality:

$$
\mathrm{R}\left(1+\frac{z q^{\prime \prime}(z)}{q^{\prime}(z)}\right)>\max \left\{0 ;-(k+1) \mathrm{R}\left(\frac{\mu}{\lambda}\right)\right\} \text {. }
$$

$$
\begin{aligned}
& (1-\lambda)\left[\frac{I_{k+1} f(z)-I_{k+1} f(-z)}{2 z}\right]^{\mu}+\lambda \frac{z\left\{I_{k} f(z)-I_{k} f(-z)\right\}}{I_{k+1} f(z)-I_{k+1} f(-z)}\left[\frac{I_{k+1} f(z)-I_{k+1} f(-z)}{2 z}\right]^{\mu} \text { if } f \in A \text { atisfies the following subordination: } \\
& (1-\lambda)\left[\frac{I_{k+1} f(z)-I_{k+1} f(-z)}{2 z}\right]^{\mu}+\lambda \frac{z\left\{I_{k} f(z)-I_{k} f(-z)\right\}}{I_{k+1} f(z)-I_{k+1} f(-z)}\left[\frac{I_{k+1} f(z)-I_{k+1} f(-z)}{2 z}\right]^{\mu} \prec q(z)+\frac{\lambda}{\mu(k+1)} z q^{\prime}(z),
\end{aligned}
$$

then

$$
\left[\frac{I_{k+1} f(z)-I_{k+1} f(-z)}{2 z}\right]^{\mu} \prec q(z), \quad(z \in U) \text {. }
$$

and $q(z)$ is the best dominant.

Proof. Let the function $h(z)$ be defined by (10). We know that the first part of (11) holds true. Combining (11) and (14), we have

$$
h(z)+\frac{\lambda}{\mu(k+1)} z h^{\prime}(z) \prec q(z)+\frac{\lambda}{\mu(k+1)} z q^{\prime}(z) .
$$

By using Lemma 2.3 and (7), we easily get the assertion of Theorem 3.2.

Corollary 3.3 Let $\lambda \in \mathrm{C}^{*}$ and $-1 \leq B<A \leq 1$. Suppose also that

$$
\mathrm{R}\left(\frac{1-B z}{1+B z}\right)>\max \left\{0 ;-(k+1) \mathrm{R}\left(\frac{\mu}{\lambda}\right)\right\} \text {. }
$$

If $f \in \mathrm{A}$ satisfies the following subordination:

$(1-\lambda)\left[\frac{I_{k+1} f(z)-I_{k+1} f(-z)}{2 z}\right]^{\mu}+\lambda \frac{z\left\{I_{k} f(z)-I_{k} f(-z)\right\}}{I_{k+1} f(z)-I_{k+1} f(-z)}\left[\frac{I_{k+1} f(z)-I_{k+1} f(-z)}{2 z}\right]^{\mu}$ $\prec \frac{1+A z}{1+B z}+\lambda \frac{(A-B) z}{(1+B z)^{2}}$, then
$\left[\frac{I_{k+1} f(z)-I_{k+1} f(-z)}{2 z}\right]^{\mu} \prec \frac{1+A z}{1+B z}, \quad(z \in U)$.

and $\frac{1+A z}{1+B z}$ is the best dominant.

If $f$ is subordinate to $F$, then $F$ is superordinate to $f$. We now derive the following superordination result for the class $\mathrm{B}^{\lambda, \mu}(A, B)$.

Theorem 3.4 let $q(z)$ be convex univalent function in $\mathrm{U}$ and let $\lambda \in \mathrm{C}$ with $\mathrm{R} \lambda>0$. Also let

$\left[\frac{I_{k+1} f(z)-I_{k+1} f(-z)}{2 z}\right]^{\mu} \in \mathrm{H}[q(0), 1] \cap \mathrm{Q}, \quad$ and

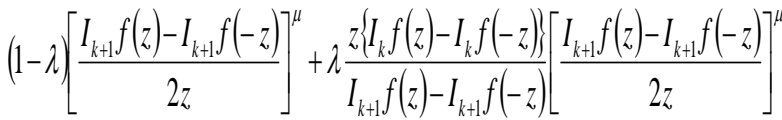

be univalent in $U$. If

$$
\begin{aligned}
& q(z)+\frac{\lambda}{\mu(k+1)} z q^{\prime}(z) \\
& \prec(1-\lambda)\left[\frac{I_{k+1} f(z)-I_{k+1} f(-z)}{2 z}\right]^{\mu}+\lambda \frac{z\left[I_{k} f(z)-I_{k} f(-z)\right)}{I_{k+1} f(z)-I_{k+1} f(-z)}\left[\frac{I_{k+1} f(z)-I_{k+1} f(-z)}{2 z}\right]^{\mu} \\
& \text { then }
\end{aligned}
$$




\section{International Journal of Science and Research (IJSR) \\ ISSN (Online): 2319-7064}

Index Copernicus Value (2013): 6.14 | Impact Factor (2015): 6.391

$q(z) \prec\left[\frac{I_{k+1} f(z)-I_{k+1} f(-z)}{2 z}\right]^{\mu}$

and $q$ is the best subordinant.

Proof. Let the function $h(z)$ be defined by (10). Then

$q(z)+\frac{\lambda}{\mu(k+1)} z q^{\prime}(z)$

$\prec(1-\lambda)\left[\frac{I_{k+1} f(z)-I_{k+1} f(-z)}{2 z}\right]^{\mu}+\lambda \frac{z\left\{I_{k} f(z)-I_{k} f(-z)\right)}{I_{k+1} f(z)-I_{k+1} f(-z)}\left[\frac{I_{k+1} f(z)-I_{k+1} f(-z)}{2 z}\right]^{\mu}$

$=h(z)+\frac{\lambda}{\mu(k+1)} z h^{\prime}(z)$.

An application of Lemma 2.4 yields the assertion of Theorem 3.4.

Taking $q(z)=\frac{1+A z}{1+B z}$ in Theorem 3.4, we obtain the following corollary.

Corollary 3.5 let $q(z)$ be convex univalent function in $\mathrm{U}$ and let $-1 \leq B<A \leq 1, \lambda \in \mathrm{C}$ with $\mathrm{R} \lambda>0$. Also let $0 \neq\left[\frac{I_{k+1} f(z)-I_{k+1} f(-z)}{2 z}\right]^{\mu} \in \mathrm{H}[q(0), 1] \cap Q$,

and

$\left(1-\lambda\left[\frac{I_{k+1} f(z)-I_{k+1} f(-z)}{2 z}\right]^{\mu}+\lambda \frac{z\left\{I_{k} f(z)-I_{k} f(-z)\right\}}{I_{k+1} f(z)-I_{k+1} f(-z)}\left[\frac{I_{k+1} f(z)-I_{k+1} f(-z)}{2 z}\right]^{\mu}\right.$ be univalent in $\mathrm{U}$. If

$\frac{1+A z}{1+B z}+\lambda \frac{(A-B) z}{(1+B z)^{2}}$

$\prec(1-\lambda)\left[\frac{\left[I_{k+1} f(z)-I_{k+1} f(-z)\right.}{2 z}\right]^{\mu}+\lambda \frac{z\left\{I_{k} f(z)-I_{k} f(-z)\right.}{I_{k+1} f(z)-I_{k+1} f(-z)}\left\{\left[\frac{I_{k+1} f(z)-I_{k+1} f(-z)}{2 z}\right]^{\mu}\right.$,

then

$\frac{1+A z}{1+B z} \prec\left[\frac{I_{k+1} f(z)-I_{k+1} f(-z)}{2 z}\right]^{\mu},(z \in U)$.

and $\frac{1+A z}{1+B z}$ is the best subordinant.

Combining the above results of subordination and superordination, we easily get the following sandwich- type result.

Corollary 3.6 let $q_{1}$ be convex univalent and let $q_{2}$ be univalent in $\mathrm{U}, \lambda \in \mathrm{C}$ with $\mathrm{R} \lambda>0$. Let $q_{2} \operatorname{satisfy(12).}$ If

$0 \neq\left[\frac{I_{k+1} f(z)-I_{k+1} f(-z)}{2 z}\right]^{\mu} \in \mathrm{H}[q(0), 1] \cap Q$,

and

$(1-\lambda)\left[\frac{I_{k+1} f(z)-I_{k+1} f(-z)}{2 z}\right]^{\mu}+\lambda \frac{z\left\{I_{k} f(z)-I_{k} f(-z)\right\}}{I_{k+1} f(z)-I_{k+1} f(-z)}\left[\frac{I_{k+1} f(z)-I_{k+1} f(-z)}{2 z}\right]^{\mu}$ be univalent function in $U$, also

$q_{1}(z)+\frac{\lambda}{\mu(n+1)} z q_{1}^{\prime}(z)$

$\prec(1-\lambda)\left[\frac{I_{k+1} f(z)-I_{k+1} f(-z)}{2 z}\right]^{\mu}+\lambda \frac{z\left\{I_{k} f(z)-I_{k} f(-z)\right\}}{I_{k+1} f(z)-I_{k+1} f(-z)}\left[\frac{I_{k+1} f(z)-I_{k+1} f(-z)}{2 z}\right]^{\mu}$

$q_{2}(z)+\frac{\lambda}{\mu(k+1)} z q_{2}^{\prime}(z)$

then

$q_{1}(z) \prec\left[\frac{I_{k+1} f(z)-I_{k+1} f(-z)}{2 z}\right]^{\mu} \prec q_{2}(z)$,

and $q_{1}$ and $q_{2}$ are, respectively, the best subordinant and dominant.

Theorem 3.7 If $\lambda \in \mathrm{C} . \mu>0$ and $f(z) \in B^{0, \mu}(1-2 \rho,-1)$, $(0 \leq \rho<1)$, then $f(z) \in \mathrm{B}^{\lambda, \mu}(1-2 \rho,-1)$ for $|z|<R$, where

$$
R=\left[\left(\sqrt{\left(\frac{|\lambda|}{\mu(k+1)}\right)^{2}}+1\right)-\frac{|\lambda|}{\mu(k+1)}\right]
$$

The bound $R$ is the best possible.

Proof. Set

$\left[\frac{I_{k+1} f(z)-I_{k+1} f(-z)}{2 z}\right]^{\mu}=(1-\rho) h(z)+\rho, \quad z \in \mathrm{U}, \quad(0 \leq \rho<1)$.

Then, clearly the function $h(z)$ is analytic in $U$ with $h(0)=1$. Proceeding as an Theorem 3.1, we have

$$
\frac{1}{1-\rho}\left\{(1-\lambda)\left[\frac{I_{k+1} f(z)-I_{k+1} f(-z)}{2 z}\right]^{\mu}\right.
$$

$$
\begin{gathered}
\left.+\lambda \frac{z\left\{I_{k} f(z)-I_{k} f(-z)\right\}}{I_{k+1} f(z)-I_{k+1} f(-z)}\left[\frac{I_{k+1} f(z)-I_{k+1} f(-z)}{2 z}\right]^{\mu}-\rho\right\} \\
=h(z)+\frac{\lambda}{\mu(k+1)} z h^{\prime}(z) .
\end{gathered}
$$

Using the following well-known estimate, see [11]

$$
\frac{\left|z h^{\prime}\right|}{\mathrm{R}[h(z)]} \leq \frac{2 r}{1-r^{2}} \quad(|z|=r<1)
$$

in (18), we obtain that

$$
\begin{aligned}
& \mathrm{R} \frac{1}{1-\rho}\left\{(1-\lambda)\left[\frac{I_{k+1} f(z)-I_{k+1} f(-z)}{2 z}\right]^{\mu}\right. \\
& \left.+\lambda \frac{z\left\{I_{k} f(z)-I_{k} f(-z)\right\}}{I_{k+1} f(z)-I_{k+1} f(-z)}\left[\frac{I_{k+1} f(z)-I_{k+1} f(-z)}{2 z}\right]^{\mu}-\rho\right\} \\
& \geq \mathrm{R} \quad h(z)\left\{1-\frac{2|\lambda| r}{\mu(k+1)\left(1-r^{2}\right)}\right\} .
\end{aligned}
$$




\section{International Journal of Science and Research (IJSR) \\ ISSN (Online): 2319-7064}

Index Copernicus Value (2013): 6.14 | Impact Factor (2015): 6.391

Right hand side of (19) is positive, provided that $r<R$, where $R$ is given by (16).

In order to show that the bound $\mathrm{R}$ is best possible, we consider the function $f(z) \in \mathrm{A}$ defined by

$$
\left[\frac{I_{k+1} f(z)-I_{k+1} f(-z)}{2 z}\right]^{\mu}=\rho+(1-\rho) \frac{1+z}{1-z} \quad(z \in \mathrm{U}, \quad 0 \leq \rho<1) \text {. }
$$

We note that

$\frac{1}{1-\rho}\left\{(1-\lambda)\left[\frac{I_{k+1} f(z)-I_{k+1} f(-z)}{2 z}\right]^{\mu}\right.$

$\left.+\lambda \frac{z\left\{I_{k} f(z)-I_{k} f(-z)\right\}}{I_{k+1} f(z)-I_{k+1} f(-z)}\left[\frac{I_{k+1} f(z)-I_{k+1} f(-z)}{2 z}\right]^{\mu}-\rho\right\}$

$=\frac{1+z}{1-z}+\frac{2|\lambda| z}{\mu(k+1)(1-z)^{2}}=0$,

for $|z|=R$, we conclude that the bound is the best possible and this proves the theorem.

Theorem 3.8 If $0 \leq \lambda_{1} \leq \lambda_{2}$ and $-1 \leq B_{1} \leq B_{2}<A_{2} \leq A_{1} \leq 1$. Then

$$
\mathrm{B}^{\lambda_{2}, \mu}\left(A_{2}, B_{2}\right) \subset \mathrm{B}^{\lambda_{1}, \mu}\left(A_{1}, B_{1}\right) \text {. }
$$

Proof. Suppose that $f \in \mathrm{B}^{\lambda_{2}, \mu}\left(A_{2}, B_{2}\right)$. We know that $\left(1-\lambda_{2}\left[\frac{I_{k+1} f(z)-I_{k+1} f(-z)}{2 z}\right]^{\mu}+\lambda_{2} \frac{z\left\{I_{k} f(z)-I_{k} f(-z)\right\}}{I_{k+1} f(z)-I_{k+1} f(-z)}\left[\frac{I_{k+1} f(z)-I_{k+1} f(-z)}{2 z}\right]^{\mu}\right.$

$$
\prec \frac{1+A_{2} z}{1+B_{2} z} \text {. }
$$

Since $-1 \leq B_{1} \leq B_{2}<A_{2} \leq A_{1} \leq 1$, we easily find that

$$
\begin{gathered}
\left(1-\lambda_{2}\right)\left[\frac{\left[I_{k+1} f(z)-I_{k+1} f(-z)\right.}{2 z}\right]^{\mu}+\lambda_{2} \frac{z\left\{I_{k} f(z)-I_{k} f(-z)\right\}}{I_{k+1} f(z)-I_{k+1} f(-z)}\left[\frac{I_{k+1} f(z)-I_{k+1} f(-z)}{2 z}\right]^{\mu} \\
\prec \frac{1+A_{2} z}{1+B_{2} z} \prec \frac{1+A_{1} z}{1+B_{1} z},
\end{gathered}
$$

this is $f \in \mathrm{B}^{\lambda_{1}, \mu}\left(A_{1}, B_{1}\right)$. Thus the assertion (20) holds true for $0 \leq \lambda_{1}=\lambda_{2}$. If $\lambda_{2}>\lambda_{1}$, by Theorem 3.1 and (21), we know that, $f \in \mathrm{B}^{0, \mu}\left(A_{2}, B_{2}\right)$ that is,

$$
\left[\frac{I_{k+1} f(z)-I_{k+1} f(-z)}{2 z}\right]^{\mu} \prec \frac{1+A_{1} z}{1+B_{1} z} .
$$

At the same time, we have

$$
\begin{aligned}
& \left(1-\lambda_{1}\right)\left[\frac{I_{k+1} f(z)-I_{k+1} f(-z)}{2 z}\right]^{\mu}+\lambda_{1} \frac{z\left\{I_{k} f(z)-I_{k} f(-z)\right)}{I_{k+1} f(z)-I_{k+1} f(-z)}\left[\frac{I_{k+1} f(z)-I_{k+1} f(-z)}{2 z}\right]^{\mu} \\
& =\frac{\lambda_{1}}{\lambda_{2}}\left\{(1-\lambda)\left[\frac{I_{k+1} f(z)-I_{k+1} f(-z)}{2 z}\right]^{\mu}+\lambda_{2} \frac{z\left\{I_{k} f(z)-I_{k} f(-z)\right\}}{I_{k+1} f(z)-I_{k+1} f(-z)}\right.
\end{aligned}
$$

$$
\left.\left[\frac{I_{k+1} f(z)-I_{k+1} f(-z)}{2 z}\right]^{\mu}\right\}+\left(1-\frac{\lambda_{1}}{\lambda_{2}}\right)\left[\frac{I_{k+1} f(z)-I_{k+1} f(-z)}{2 z}\right]^{\mu}
$$

Moreover,

$0 \leq \frac{\lambda_{1}}{\lambda_{2}}<1$

and the function $\frac{1+A_{1} z}{1+B_{1} z},-1 \leq B_{1}<A_{1} \leq 1, z \in \mathrm{U}$ is analytic and convex in $U$. Combining (21 - 23) and Lemma 2.4 , we find that

$\left(1-\lambda_{1}\right)\left[\frac{I_{k+1} f(z)-I_{k+1} f(-z)}{2 z}\right]^{\mu}+\lambda_{1} \frac{z\left\{I_{k} f(z)-I_{k} f(-z)\right\}}{I_{k+1} f(z)-I_{k+1} f(-z)}\left[\frac{I_{k+1} f(z)-I_{k+1} f(-z)}{2 z}\right]^{\mu}$ $\prec \frac{1+A_{1} z}{1+B_{1} z}$.

this is $f \in \mathrm{B}^{\lambda_{1}, \mu}\left(A_{1}, B_{1}\right)$, which implies that the assertion (12) of Theorem 3.8 holds and this completes the proof.

Theorem 3.9 Let $f(z) \in \mathrm{B}^{\lambda, \mu}(A, B)$ with $\lambda>0$ and $-1 \leq B_{1}<A_{1} \leq 1$. Then

$\frac{\mu(k+1)}{\lambda} \int_{0}^{1} \frac{1-A u}{1-B u} u^{\frac{\mu(k+1)}{\lambda}-1} d u$

$<\mathrm{R}\left[\frac{I_{k+1} f(z)-I_{k+1} f(-z)}{2 z}\right]^{\mu}<\frac{\mu(k+1)}{\lambda} \int_{0}^{1} \frac{1+A u}{1+B u} u^{\frac{\mu(k+1)}{\lambda}-1} d u$

The extremal function of (24) is defined by

$F_{\lambda, \mu, A, B}(z)=2 z\left(\frac{\mu(k+1)}{\lambda} \int_{0}^{1} \frac{1+A z u}{1+B z u} u^{\frac{\mu(k+1)}{\lambda}-1} d u\right)^{\frac{1}{\mu}}$.

Proof. Let $f(z) \in \mathrm{B}^{\lambda, \mu}(A, B)$ with $\lambda>0$. From

Theorem 1, we know that (1) holds, which implies that

$\mathrm{R}\left[\frac{I_{k+1} f(z)-I_{k+1} f(-z)}{2 z}\right]^{\mu}<\sup _{z \in U} \mathrm{R}\left\{\frac{\mu(k+1)}{\lambda} \int_{0}^{1} \frac{1+A z u}{1+B z u} u^{\frac{\mu(k+1)}{\lambda}-1} d u\right\}$

$\leq\left\{\frac{\mu(k+1)}{\lambda} \int_{0}^{1} \sup _{z \in U} \mathrm{R}\left(\frac{1+A z u}{1+B z u}\right) u^{\frac{\mu(k+1)}{\lambda}-1} d u\right\}$

$<\frac{\mu(k+1)}{\lambda} \int_{0}^{1} \frac{1+A u}{1+B u} u^{\frac{\mu(k+1)}{\lambda}-1} d u$,

and

$\mathrm{R}\left[\frac{I_{k+1} f(z)-I_{k+1} f(-z)}{2 z}\right]^{\mu}>\inf _{z \in U} \mathrm{R}\left\{\frac{\mu(k+1)}{\lambda} \int_{0}^{1} \frac{1+A z u}{1+B z u} u^{\frac{\mu(n+1)}{\lambda}-1} d u\right\}$ $\geq\left\{\frac{\mu(k+1)}{\lambda} \int_{0}^{1} \inf _{z \in \cup} \mathrm{R}\left(\frac{1+A z u}{1+B z u}\right) u^{\frac{\mu(k+1)}{\lambda}-1} d u\right\}$

$>\frac{\mu(k+1)}{\lambda} \int_{0}^{1} \frac{1+A u}{1+B u} u^{\frac{\mu(k+1)}{\lambda}-1} d u$.

Combining (26) and (27), we obtain (20). Noting that the function $F_{\lambda, \mu, A, B}(z)$ defined by (25) belongs to the class 


\section{International Journal of Science and Research (IJSR) \\ ISSN (Online): 2319-7064 \\ Index Copernicus Value (2013): 6.14 | Impact Factor (2015): 6.391}

$\mathrm{B}^{\lambda, \mu}(A, B)$, we get that inequality (24) is sharp. This completes the proof.

In view of Theorem 9 , we have the following distortion theorems for the class $\mathrm{B}^{\lambda, \mu}(A, B)$.

Corollary 3.10 Let $f(z) \in \mathrm{B}^{\lambda, \mu}(A, B)$ with $\lambda>0$ and $-1 \leq B_{1}<A_{1} \leq 1$. Then for $|z|=r<1$, we have

$2 r\left(\frac{\mu(k+1)}{\lambda} \int_{0}^{1} \frac{1-A u r}{1-B u r} u^{\frac{\mu(k+1)}{\lambda}-1} d u\right)^{\frac{1}{\mu}}$

$<\left|I_{k+1} f(z)-I_{k+1} f(-z)\right|<2 r\left(\frac{\mu(k+1)}{\lambda} \int_{0}^{1} \frac{1+A u r}{1+B u r} u^{\frac{\mu(k+1)}{\lambda}-1} d u\right)^{\frac{1}{\mu}}$.

The extremal function of (28) is defined by (25).

By noting that

$(\mathrm{R}(v))^{\frac{1}{2}} \leq \mathrm{R}\left(v^{\frac{1}{2}}\right) \leq|v|^{\frac{1}{2}}, \quad v \in \mathrm{C} ; \mathrm{R}(v) \geq 0$.

From Theorem 9, we can easily derive the following result.

Corollary 3.11 Let $f(z) \in \mathrm{B}^{\lambda, \mu}(A, B)$ with $\lambda>0$ and

$-1 \leq B_{1}<A_{1} \leq 1$. Then

$\left(\frac{\mu(k+1)}{\lambda} \int_{0}^{1} \frac{1-A u}{1-B u} u^{\frac{\mu(k+1)}{\lambda}-1} d u\right)^{\frac{1}{2}}$

$<\mathrm{R}\left[\frac{I_{k+1} f(z)-I_{k+1} f(-z)}{2 z}\right]^{\frac{\mu}{2}}<\left(\frac{\mu(k+1)}{\lambda} \int_{0}^{1} \frac{1+A u}{1+B u} u^{\frac{\mu(k+1)}{\lambda}-1} d u\right)^{\frac{1}{2}}$

Theorem 3.12 Let $f(z) \in \mathrm{B}^{\lambda, \mu}(A, B)$ with $\lambda>0$ and $-1 \leq B_{1}<A_{1} \leq 1$. Then

$$
\left|a_{1}\right| \leq\left|\frac{2(A-B)}{\lambda+2 \mu(k+1)}\right| .
$$

The inequality (29) is sharp, with the extremal function defined by (25).

Proof. Combining (1) and (5), we have

$(1-\lambda)\left[\frac{I_{k+1} f(z)-I_{k+1} f(-z)}{2 z}\right]^{\mu}+\lambda \frac{z\left\{I_{k} f(z)-I_{k} f(-z)\right\}}{I_{k+1} f(z)-I_{k+1} f(-z)}\left[\frac{I_{k+1} f(z)-I_{k+1} f(-z)}{2 z}\right]^{\mu}$

$=1+\left[1+\frac{\lambda}{2 \mu(k+1)}\right] \mu(k+1) a_{1} z+\cdots \prec \frac{1+A z}{1+B z}$

$$
=1+(A-B) z+\cdots
$$

An application of Lemma 2.4 to (30) yields

$$
\left|\left[1+\frac{\lambda}{2 \mu(k+1)}\right] \mu(k+1) a_{1}\right| \leq|A-B| .
$$

Thus, from (31), we easily arrive at (29) asserted by Theorem 3.12.
Theorem 3.13 Let $f(z) \in \mathrm{B}^{\lambda, \mu}(A, 0)$ with $\mathrm{R} \lambda>0$ and $A>0$ and $|\lambda|\left(1+\mathrm{R} \frac{\mu(k+1)}{\lambda}\right)>A \mu(k+1)$. Then

$\left|\frac{z\left\{I_{k} f(z)-I_{k} f(-z)\right\}}{I_{k+1} f(z)-I_{k+1} f(-z)}-1\right|<\frac{A\left\{\lambda\left(1+\mathrm{R}\left(\frac{\mu(k+1)}{\lambda}\right)\right)+\mu(k+1)\right\}}{|\lambda|\left\{|\lambda|\left(1+\mathrm{R}\left(\frac{\mu(k+1)}{\lambda}\right)\right)-A \mu(k+1)\right\}}$ Proof. let $h(z)$ be defined by (9). It follows from (10) that $h(z)+\frac{\lambda}{\mu(k+1)} z h^{\prime}(z)=1+A w(z)$,

where

$$
w(z)=\sum_{n=1}^{\infty} w_{n} z_{n},
$$

is analytic in $\mathrm{U}$ with $|w(z)|<1, z \in \mathrm{U}$. From (32), we can get

$$
\begin{aligned}
& h(z)=1+A \frac{\mu(k+1)}{\lambda} \int_{0}^{1} t^{\frac{\mu(k+1)}{\lambda}-1} w(t z) d t \\
& =1+A \frac{\mu(k+1)}{\lambda} \sum_{n=1}^{\infty} \frac{1}{n+\frac{\mu(k+1)}{\lambda}} w_{n} z^{n} .
\end{aligned}
$$

It follows from (33) that

$$
\begin{aligned}
& (z h(z))^{\prime}=1+A \frac{\mu(k+1)}{\lambda} \sum_{n=1}^{\infty} \frac{n+1}{n+\frac{\mu(k+1)}{\lambda}} w_{n} z^{n} . \\
& =1+A \frac{\mu(k+1)}{\lambda} \sum_{n=1}^{\infty} \frac{1}{n+\frac{\mu(k+1)}{\lambda}} w_{n} z^{n} \\
& +A \frac{\mu(k+1)}{\lambda}\left(w(z)-\frac{\mu(k+1)}{\lambda} \int_{0}^{1} t^{\frac{\mu(k+1)}{\lambda}-1} w(t z) d t\right) .
\end{aligned}
$$

We now find from (33) and (34) that

$(z h(z))^{\prime}=A \frac{\mu(k+1)}{\lambda}\left(w(z)-\frac{\mu(k+1)}{\lambda} \int_{0}^{1} t^{\frac{\mu(k+1)}{\lambda}-1} w(t z) d t\right)$

Combining (33) and (35), we can get

$$
\left|\frac{z h^{\prime}(z)}{h(z)}\right|<\frac{A\left\{\mid \lambda\left(1+\mathrm{R}\left(\frac{\mu(k+1)}{\lambda}\right)\right)+\mu(k+1)\right\}}{|\lambda|\left\{|\lambda|\left(1+\mathrm{R}\left(\frac{\mu(k+1)}{\lambda}\right)\right)-A \mu(k+1)\right\}}
$$

Thus, from (10) and (36), we easily arrive at the assertion of Theorem 3.13 .

Remark 3.1 If $\mu=1$, we obtain the results of [2], Theorems 3.1, 4.1 and 4.4 . 


\section{References}

[1] Ali Muhammad, Some differential subordination and superordinations properties of symmetric functions, Rend. Sem. Mat. Univ. Politec. Torino 69 ,3, 247- 259, 2011.

[2] Ali Muhammad and Amjadullak Khattak, Some differential subordination and superordination properties of symmetric analytic functions involving Noor integral operator, Le Mathamatiche Vol. 67 (2012) Fasc. II, 77- 92.

[3] M. K. Aouf- T. Bulboac $\breve{a}$, Subordination and superordination properties of multivalent functions defined by certain integral operator, J. Franklin Inst, 347, 641-653, 2010.

[4] M. K. Aouf- F. M. Al-Oboudi- M. M. Haidan, On some results for $\lambda$-spirallike and $\lambda$-Robertson functions of complex order, Publ. Inst. Math. Belgrade 77, 91, 9398, 2005.

[5] M. K. Aouf - T. M. Seoudy, Some properties of a class of multivalent analytic functions involving the LiuOwa operator, J. Com. Math. App. 60, 1525- 1535, 2010.

[6] T. Bulboac $\breve{a}$, A class of superordination preserving integral opeators, Indag. Math. (New Ser.) 13, 3, 301311, 2002.

[7] T. Bulboac $\breve{a}$, Classes of first-order differential subordination, Demonstratio Math. 35, 2, 287292,2002.

[8] J. L. Liu, The Noor integral and strongly starlike functions, J. Math. Anal. Appl. 261, 441- 447, 2001.

[9] J. L. Liu - K. I. Noor, Some properties of Noor integral operator, J. Nat. Geom. 21, 81- 90, 2002.

[10] M. S. Liu, On certain subclass of analytic functions, J. South China Normal Univ. 4, 15- 20, 2002.

[11] T. H. Macgregor, The radius of univalence of certain analytic functions, Proc. Amer. Math. Soc. 14, 514520, 1963.

[12] S. S. Miller - P. T. Mocanu, Differential subordination Theory and Applications, Series on Monographs and Textbooks in Pure and Applied Mathematics, vol. 225, Marcel Dekker Inc., New York, Basel, 2000.

[13] S. S. Miller- P. T. Mocanu, Subordinations of differential superordinations, Complex Variables 48, 10, 815-826, 2003.

[14] K. I. Noor, On new classes of integral operators, J. Natur. Geom. 16, 71- 80, 1999.

[15] K. I. Noor - M. A. Noor, On integral operators, J. Math. Anal. Appl. 238, 341- 352, 1999.

[16] W. Rogosinski, On the coefficient of subordinate functions, Proc. Lond. Math. Soc. Ser.2 48, 48- 82, 1943.

[17] S. Ruscheweyh, New criteria for univalent functions, Proc. Amer. Math. Soc. 49, 109- 115, 1975.

[18] K. Sakaguchi, On a certain univalent mapping, J. Math. Soc. Japan, 11, 72- 75, 1959.

[19] T. N. Shanmugam, V. Ravichandran and S. Sivasubbramanian, Differential sandwich theorems for subclasses of analytic functions, Aust. J. Math. Anal. Appl. 3, 1- 11, Art. 8, 2006. 